\title{
Valproic acid inhibits epithelial-mesenchymal transition in renal cell carcinoma by decreasing SMAD4 expression
}

\author{
SHAOWEI MAO ${ }^{1}$, GUOLIANG LU ${ }^{1}$, XIAOPENG LAN ${ }^{1}$, CHUANWEI YUAN $^{1}$, \\ WEI JIANG ${ }^{2}$, YOUGEN CHEN ${ }^{1}$, XUNBO JIN $^{1}$ and QINGHUA XIA ${ }^{1}$ \\ ${ }^{1}$ Minimally Invasive Urology Center, Shandong Provincial Hospital Affiliated to Shandong University, Jinan, \\ Shandong 250014; ${ }^{2}$ Department of Urology, Dongying People's Hospital, Dongying, Shandong 257000, P.R. China
}

Received September 21, 2016; Accepted June 27, 2017

DOI: $10.3892 / \mathrm{mmr} .2017 .7394$

\begin{abstract}
Renal cell carcinoma (RCC) is the most common malignancy in urogenital neoplasms worldwide. According to previous studies, valproic acid (VPA), an anticonvulsant drug, can suppress tumor metastasis and decrease the expression level of Mothers against decapentaplegic homolog 4 (SMAD4) and therefore may inhibit epithelial-mesenchymal transition (EMT), which is responsible for cancer metastasis. However, the association between VPA, EMT and SMAD4 in RCC metastasis remains obscure. In the present study, it was demonstrated that in the RCC cell lines 786-O and Caki-1 treated with VPA, the neural (N)-cadherin, vimentin and SMAD4 protein and mRNA levels were decreased, accompanied with an increase in expression of epithelial (E)-cadherin. Silencing SMAD4 expression decreased the expression of EMT markers, including $\mathrm{N}$-cadherin and simultaneously upregulated E-cadherin in RCC cell lines. SMAD4 overexpression counteracted the VPA-mediated EMT-inhibitory effect $(\mathrm{P}<0.05)$. The present study demonstrates that VPA inhibited EMT in RCC cells via altering SMAD4 expression. In addition, immunohistochemical staining demonstrated that transforming growth factor- $\beta$ (TGF- $\beta$ ) and low expression of SMAD4 was associated with a lower Fuhrman grade and low expression of transcription intermediary factor $1-\gamma$ was associated with a higher tumor Fuhrman grade $(\mathrm{P}<0.05)$, Therefore, based on the regulatory effect of SMAD4 on EMT-associated transcription factors, SMAD4 which can form a SMAD3/SMAD4 complex induced by TGF- $\beta$, could be a potential anticancer drug target inhibiting tumor invasion and metastasis in RCC.
\end{abstract}

Correspondence to: Dr Qinghua Xia, Minimally Invasive Urology Center, Shandong Provincial Hospital Affiliated to Shandong University, 9677 Jingshidong Road, Jinan, Shandong 250014, P.R. China

E-mail: finy2940@sina.com

Key words: mothers against decapentaplegic homolog 4 , transcription intermediary factor $1-\gamma$, valproic acid, epithelial-mesenchymal transition, renal cell carcinoma

\section{Introduction}

Accounting for 2-3\% of all human malignancies, renal cell carcinoma (RCC) is the most common kidney malignancy worldwide, with clear-cell RCC (ccRCC) being the most common subtype (1). The incidence of RCC continues to increase and in 2012, there were $>338,000$ novel cases and 143,000 individuals succumbed to kidney cancer worldwide (2). Despite advances in diagnosis and treatment, particularly improved imaging techniques, patients with $\mathrm{RCC}$ have a poor prognosis, making RCC a serious problem for oncological healthcare around the world (3). The 5-year cancer-specific survival rate of metastatic ccRCC is $<27.1 \%$, decreased compared with that of nonmetastatic ccRCC, which is $70 \%$, and ccRCC is prone to metastasis (4). The mechanism of cancer metastasis and the cause of resistance to treatment are currently poorly understood, and deserve more attention and study.

The epithelial-mesenchymal transition (EMT), a process that transforms epithelial cell phenotypes into mesenchymal ones, has been demonstrated to serve a pivotal role in numerous steps of metastatic progression in tumors. In EMT, epithelial cells lose polarity, disassemble cell-cell junctions and gain more mesenchymal and motile properties (5), which provides cancer cells with a greater capacity to invade and disseminate to distant sites. This phenomenon is triggered by a series of complex and multi-layered growth factors recruited from the tumor microenvironments (6).

Transforming growth factor- $\beta$ (TGF- $\beta$ ) is a pluripotent cytokine with divergent roles in cancer progression (7). The factor that determines whether TGF- $\beta$ acts as a tumor suppressor or promoter has been the subject of study. Several transcription factors, including zinc-finger proteins SNAI1 and SNAI2, zinc finger E-box-binding homeobox 1 and 2, and Twist-related protein 1 have been defined as initiators of EMT (8). TGF- $\beta$ has also been reported to serve a crucial role in initiating EMT in various types of cancer (5). However, the understanding of the function of early response transcription factors remains unclear. The Mothers against decapentaplegic homolog 4 (SMAD4) protein is recognized as a central mediator of TGF- $\beta$ and/or bone morphogenetic protein signaling pathways (9). A recent study has demonstrated that the loss of SMAD4 leads to the dysfunction of the canonical TGF- $\beta$ signaling pathway. However, in numerous types of cancer 
TGF- $\beta$ switches from tumor suppressor to tumor promoter, thereby driving invasion and metastasis (10). By activating SMAD-dependent and independent pathways, TGF $\beta$ acts as an inducer of EMT (11), and SMAD4 has been considered to be an independent prognostic factor in ccRCC (12).

Epigenetic modification including histone acetylation is one mechanism for controlling gene expression. Histone acetylation is mediated by the counteracting activity of histone acetyltransferases and histone deacetylases (HDACs). The reversible acetylation and deacetylation of histones is always accompanied by the activation and silencing of gene expression (13). Histone post-translational modifications (PTMs) serve a fundamental role in the control of processes involving the DNA template within the cell. Although a number of PTMs mediate their effects through histone-histone or histone-DNA interaction, a large fraction function through the recruitment of chromatin-associated proteins that harbor conserved 'reader' domains (14).

Valproic acid (VPA), a classic anticonvulsant drug used for decades, has previously been demonstrated to be a potent class I HDAC inhibitor (15). VPA may induce several anticancer effects, particularly the inhibition of cancer cell proliferation, growth and differentiation (16). Cell cycle, growth and apoptosis were also influenced by VPA in RCC (17).

The present study presents the evidence that VPA negatively regulates SMAD4 expression in RCC cells, thereby inhibiting cancer cell metastasis and invasion. The aim was to identify the role served by SMAD4 in RCC progression and metastasis by administering VPA to RCC cell lines, and analyzing the association between VPA, SMAD4 and EMT regulation.

\section{Materials and methods}

Cell culture and reagents. Caki-1, 786-O and HK-2 cells were used in the present study and were purchased from the China Center for Type Culture Collection (Wuhan, China). According to the American Type Culture Collection, Caki-1 is a metastatic RCC cell line that demonstrates highly invasive behavior, 786-O is a non-metastatic RCC cell line and HK-2 is a normal renal cell line. Caki-1 cells were cultured in Mac5a media (GIBCO; Thermo Fisher Scientific, Inc., Waltham, MA, USA) and 786-O cells were cultured in RPMI-1640 media (GIBCO; Thermo Fisher Scientific, Inc.), HK-2 cells were cultured in DMEM/F12 media (GIBCO; Thermo Fisher Scientific, Inc.). All cell lines were cultured in medium supplemented with $10 \%$ fetal bovine serum (FBS; Biological Industries, Kibbutz Beit Haemek, Israel) and $1 \%$ penicillin/streptomycin at $37^{\circ} \mathrm{C}$ under $5 \% \mathrm{CO}_{2}$. VPA (Sigma-Aldrich; Merck KGaA, Darmstadt, Germany) was diluted to a concentration of $50 \mathrm{mmol} / 1$ and stored at $-20^{\circ} \mathrm{C}$. VPA was dissolved in medium at concentrations of 1.2, 2.4 and $5 \mathrm{mmol} / \mathrm{l}$. Caki-1 and 786-O cells were treated in a dose- $(1.2,2.4$ and $5 \mathrm{mmol} / 1$ for $48 \mathrm{~h}$ ) and time-dependent manner $(12,24,48 \mathrm{~h}$ at $2.4 \mathrm{mmol} / \mathrm{l})$, and cells in the control groups were incubated with medium alone.

Plasmids and transfection. Genechem-GV230 and Genechem-GV248 plasmids, which express full-length human SMAD4 and short hairpin RNA (shRNA) against SMAD4, respectively, were provided by Shanghai GeneChem Co., Ltd., (Shanghai, China). Negative control plasmids (cat. no. BCON0831407400) were also purchased from Shanghai GeneChem Co., Ltd (Shanghai, China). Transfection of 786-O cells with these plasmids was performed using Lipofectamine ${ }^{\circledR} 2000$ in vitro transfection reagent (Thermo Fisher Scientific, Inc.); $1 \mu \mathrm{g}$ plasmid was added per well of 6 -well plate $\left(1 \times 10^{4}\right.$ cells/well). After $10 \mathrm{~h}$ transfection, the medium was changed to complete RPMI-1640 media. Then, following culture for $48 \mathrm{~h}$, the cells were treated by VPA for $48 \mathrm{~h}$. The transfection efficiency was detected by fluorescence microscopy.

Antibodies. Mouse anti-SMAD4 (cat. no. sc-7966; 1:500) and mouse anti- $\beta$-actin (cat. no. sc-130301; 1:1,000) were obtained from Santa Cruz Biotechnology, Inc., (Dallas, TX, USA). Mouse anti-epithelial (E)-cadherin (cat. no. 610181; 1:1,000), mouse anti-neural (N)-cadherin (cat. no. 610920, 1:500), and mouse anti-vimentin (cat. no. 550513; 1:1,000 dilution) antibodies were purchased from BD Biosciences (Franklin lakes, NJ, USA). Horseradish peroxidase (HRP)-conjugated goat anti-mouse immunoglobulin $\mathrm{G}$ (heavy and light chains) secondary antibodies (cat. no. A0216) were acquired from Beyotime Institute of Biotechnology (Haimen, China).

Western blot analysis. Cell lysates were prepared from transfected cells and from VPA-treated cells following lysis in RIPA Lysis and Extraction Buffer (Thermo Fisher Scientific, Inc.). The protein concentrations were detected using the BCA Protein Assay kit (23235; Thermo Fisher Scientific, Inc.) Then, $40 \mu \mathrm{g}$ of total proteins were separated by $10 \%$ SDS-PAGE and transferred onto a PVDF membrane (EMD Millipore, Billerica, MA, USA). Following blocking with 5\% milk in TBST for $60 \mathrm{~min}$ at room temperature and probing with protein-specific antibodies at $4^{\circ} \mathrm{C}$ overnight, the membranes were incubated with secondary antibodies diluted in TBS-Tween-20 (0.075\%) for $1 \mathrm{~h}$ at room temperature. The blots were detected with Chemiluminescent HRP Substrate (EMD Millipore) and visualized using an LAS-4000 Luminescent Image Analyzer (Fujifim Corporation, Tokyo, Japan). The densitometry was assessed using ImageJ version $1.49 \mathrm{~V}$ (National Institutes of Health, Bethesda, MD, USA) All experiments were performed in duplicate and repeated three times.

Immunohistochemical staining. A total of 39 cancer tissue samples were obtained from patients with RCC who underwent partial or radical nephrectomy at the Shandong Provincial Hospital (Shandong, China) between January 2004 and May 2015. Of those patients, 29 were male (age, 59.2 \pm 8.7 ), and 10 were female (age, 61.5 \pm 6.9 ). Samples were cut into $5 \mu \mathrm{m}$ paraffin sections, which were then deparaffinized and rehydrated. Antigen retrieval was performed by heating the sections for $2 \mathrm{~min}$ in citrate buffer at a $\mathrm{pH}$ 6.0. Endogenous peroxidase activity was blocked with $3 \% \mathrm{H}_{2} \mathrm{O}_{2}$ for $30 \mathrm{~min}$. Following subsequent blocking with $5 \%$ bovine serum albumin (BSA; A8020; Beijing Solarbio Science \& Technology Co., Ltd., Beijing, China) for $30 \mathrm{~min}$, the sections were incubated overnight at $4^{\circ} \mathrm{C}$ with primary antibodies against $\mathrm{Smad} 4$ (cat. no.SC-7966; 1:200; Santa Cruz Biotechnology,Inc.), TIF1- $\gamma$ (cat. no. SC-101179; 1:250; Santa Cruz Biotechnology, Inc.) in phosphate-buffered saline (PBS) and TGF- $\beta$ (cat. no. RAB-023B; Fuzhou Maxim (Maixin) Biotech Co., Ltd., Fuzhou, China), 
A



B

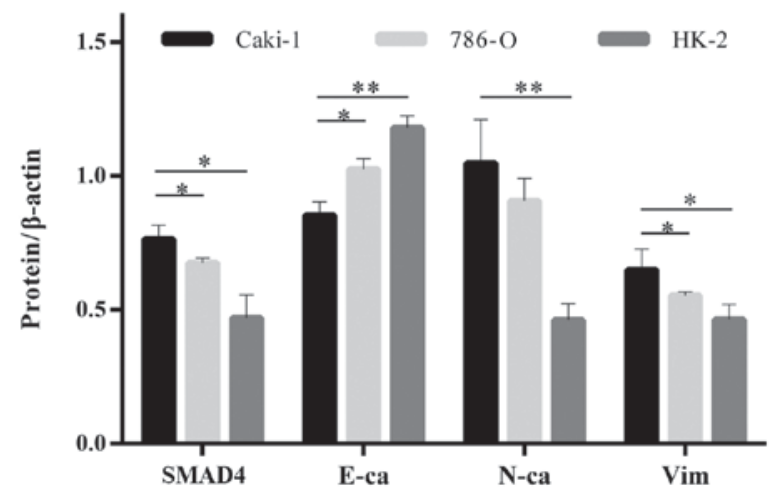

Figure 1. Expression pattern of SMAD4 and EMT markers in renal cell carcinoma cell lines and valproic acid inhibits invasion and migration in 786-O cells. (A) Western blot analysis and (B) relative expression levels of SMAD4 protein and EMT markers in Caki-1, 786-O, HK-2 cell lines. Data is demonstrated as the mean \pm standard deviation from three independent experiments. ${ }^{*} \mathrm{P}<0.05$ vs. control group, ${ }^{* *} \mathrm{P}<0.01$ vs. control group. E-ca, epithelial-cadherin; N-ca, neural-cadherin; Vim, vimentin; EMT, epithelial-mesenchymal transition; SMAD4, Mothers against decapentaplegic homolog 4

and with PBS alone as a negative control. The sections were then incubated with horseradish peroxidase (HRP)-conjugated secondary antibodies (SE13; 1:5,000; Beijing Solarbio Science \& Technology Co., Ltd.) for $20 \mathrm{~min}$ at $37^{\circ} \mathrm{C}$. HRP activity was detected using 3'3-diaminobenzidine for $1 \mathrm{~min}$. The slides were stained with haematoxylin for $5 \mathrm{~min}$ at room temperature and then dehydrated and mounted using neutral balsam., and three independent observers blindly performed the measurements (magnification, $\mathrm{x} 400$ ) using an Olympus microscope (X31-32C02; Olympus Corporation, Tokyo, Japan). The present study was approved by the Provincial Hospital Affiliated to Shandong University ethics committee (Jinan, China) and all participants provided written informed consent.

Reverse transcription-quantitative polymerase chain reaction (RT-qPCR). Total RNA from 786-O and Caki-1 cells was analyzed using RT-qPCR via two kits purchased from Takara Bio, Inc., Otsu, Japan: MiniBEST Universal RNA Extraction kit (cat.no. 9767) and PrimeScriptTM RT reagent kit (cat. no. RR037A). qPCR was performed as follows: $42^{\circ} \mathrm{C}$ for $30 \mathrm{~min}, 95^{\circ} \mathrm{C}$ for $10 \mathrm{~min}$ and followed by 40 cycles of amplification at $95^{\circ} \mathrm{C}$ for $20 \mathrm{sec}, 62^{\circ} \mathrm{C}$ for $30 \mathrm{sec}, 72^{\circ} \mathrm{C}$ for $30 \mathrm{sec}$. The following primer sequences were used: SMAD4 forward, 5'-CTTTCCCAACATTCCTGTGG-3'; reverse, 5'-ATCCAT TCTGCTGCTGTCCT-3'; E-cadherin forward 5'-AGAATG ACAACAAGCCCGAAT-3'; reverse, 5'-CGGCATTGTAGG TGTTCACA3'; N-cadherin forward GGACAGTTCCTGAGG
GATCA-3'; reverse, GGATTGCCTTCCATGTCTGT, $\beta$-actin forward, 5'-AATCCCATCACCATCTTCCA-3'; and reverse, 5'-TGGACTCCACGACGTACTCA-3'. The relative expression of SMAD4 mRNA was determined using the expression of $\beta$-actin as a reference. Relative mRNA expression change was determined using $2^{-\Delta \Delta C t}$ method (18). All experiments were performed in duplicate and repeated three times.

Migration and invasion assays. Cellular migration and invasion assays were performed using a Boyden chamber containing 24-well Transwell plates (Corning Incorporated, Corning, NY, USA) with $8-\mathrm{mm}$ pores in the membrane. For migration assays, $\sim 7.5 \times 10^{4}$ cells in $200 \mathrm{ml}$ of FBS-free medium were transferred into the upper chamber and the lower chamber was filled with complete medium (containing 10\% FBS) as a chemoattractant. Following $24 \mathrm{~h}$ of incubation at $37^{\circ} \mathrm{C}$ in a $5 \% \mathrm{CO}_{2}$ atmosphere, the membranes containing the cells were fixed with $95 \%$ alcohol in $30 \mathrm{~min}$ at room temperature and stained with $0.1 \%$ crystal violet Images were captured of the lower surfaces of the membranes at 100x magnification. Images were captured of five random fields in each chamber to determine migration. For invasion assays, the membrane was coated with $50 \mathrm{ml}$ of diluted Matrigel ${ }^{\circledR}$ (1:7; BD Biosciences). Following the solidification of the Matrigel at $37^{\circ} \mathrm{C}, 1.0 \times 10^{5}$ cells in $200 \mathrm{ml}$ of culture medium supplemented with $1 \%$ FBS were seeded into the upper chamber, whereas the lower chamber was filled with complete medium. Then, the Boyden chamber was incubated at $37^{\circ} \mathrm{C}$ with a $5 \% \mathrm{CO}_{2}$ atmosphere for $24 \mathrm{~h}$. The subsequent staining and observation procedures were identical to those of the migration assays. Quantification was performed by counting migratory cells using light microscopy in three individual fields per insert. All experiments were performed in duplicate and repeated three times.

Statistical analysis. All experiments were performed in triplicate as a minimum. The data were statistically analyzed using SPSS for Windows Statistics version 20 (IBM Corp., Armonk, NY, USA). The Kruskal-Wallis H test was used to analyze immunohistochemical-staining scores. One-way analysis of variance was used to analyze the differences of the grey level data of the protein bands, the relative mRNA expression levels, and the number of invasive and migratory cells between four groups, following that Fisher's least significant difference method was used for post hoc comparisons. $\mathrm{P}<0.05$ was considered to indicate a statistically significant difference.

\section{Results}

Profile of SMAD4 status and EMT expression in renal cells of different characteristics. Different expression patterns of SMAD4 and EMT markers were observed in Caki-1 and 786-O cell lines and therefore all three of the cell lines (Caki-1, 786-O, HK-2) of varying invasive capacities were screened for SMAD4 and EMT marker expression. SMAD4 protein was highly expressed in Caki-1 cells, which exhibited greater invasive behavior, whereas 786-O and HK-2 cells had a lower expression level of SMAD4 and mesenchymal markers (N-cadherin and vimentin) and a lower invasive ability (Fig. 1A and B). Together, these results suggest a potential correlation between EMT characteristics, SMAD4 expression and cell invasion in RCC cells. 
A

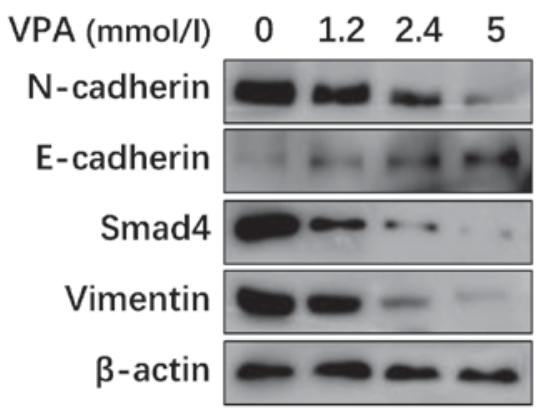

C

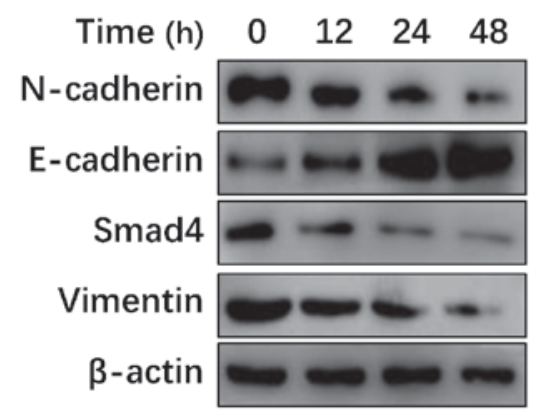

B

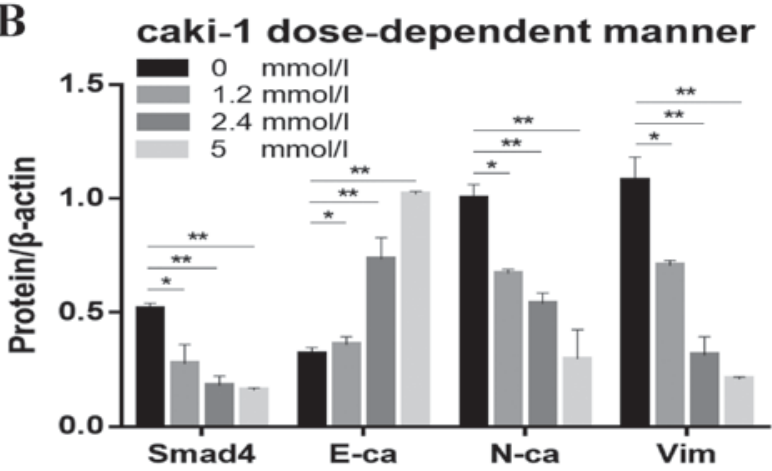

D



Figure 2. SMAD4 and EMT markers were inhibited by VPA in renal cell carcinoma cells. (A) Western blot and (B) quantified protein expression levels of SMAD4 and EMT makers treated with VPA in a dose-dependent manner (0, 1.2, 2.4 and 5 mmol/l) in Caki-1 cells. (C) Western blot and (D) quantified protein expression levels of SMAD4 and EMT markers treated with VPA in a time-dependent (0, 12, 24 and 48 h) manner in Caki-1 cells. Data is demonstrated as the mean \pm standard deviation from three independent experiments. ${ }^{*} \mathrm{P}<0.05$ vs. control group ${ }^{* *} \mathrm{P}<0.01$ vs. control group. $\mathrm{N}$-ca, neural cadherin; E-ca, epithelial cadherin; EMT, epithelial-mesenchymal transition; VPA, valproic acid; Vim, vimentin; SMAD 4, Mothers against decapentaplegic homolog 4.

VPA alters the expression of EMT markers and suppresses migration and invasion in RCC cells. The expression of SMAD4 and EMT markers in RCC cell lines was analyzed using western blotting. In Caki-1 and 786-O cells, treatment with VPA decreased expression of SMAD4 and the mesenchymal markers $\mathrm{N}$-cadherin and vimentin but increased E-cadherin expression (Figs. 2 and 3), providing more evidence of the influence of VPA on EMT. Further experiments demonstrated that the SMAD4 mRNA level was suppressed in a dose (VPA doses of 1.2, 2.4 and $5 \mathrm{mmol} / \mathrm{l}$ ) and a time-dependent manner $(0,12,24,48 \mathrm{~h})$ in the two cell lines (Fig. 4). In vitro cell invasion and migration assays were performed to study the invasive and migratory ability of 786-O cells treated with different VPA concentrations (Fig. 5). It was demonstrated that the lower and higher concentrations of VPA significantly diminished cell invasive and migratory ability in 786-O cells $(\mathrm{P}<0.05)$. Tumor cell invasion and migration ability were suppressed by VPA treatment. VPA treatment induced a transition from round-like to long-shaped morphology (Fig. 6).

SMAD4 expression regulates EMT status with or without VPA treatment. To confirm the interaction between SMAD4 and EMT status, 786-O cells were transfected with a plasmid to transiently knock down SMAD4 expression. Western blot analysis confirmed the efficiency of transfection and demonstrated near absence of SMAD4 in the knockdown 786-O cells, accompanied with significantly upregulated E-cadherin, and downregulated N-cadherin and vimentin expression (Fig. 7). Following treatment with VPA, SMAD4-knockdown cells exhibited little alteration in the expression levels of E-cadherin and $\mathrm{N}$-cadherin. Additionally, 786-O cells were transfected with a plasmid that upregulated SMAD4 and were then treated by VPA. Compared with the control, E-cadherin protein levels were significantly decreased, and $\mathrm{N}$-cadherin and vimentin expression were increased, which indicated that SMAD4 overexpression negated the inhibitory effect of VPA on EMT (Fig. 7).

SMAD4, TIF $1-\gamma$ and TGF- $\beta$ expression in vivo. To further investigate the role of SMAD4 in the TGF- $\beta$ signaling pathway, immunohistochemical staining was performed on 39 specimens to detect the expression levels of SMAD4, TGF- $\beta$ and TIF1- $\gamma$ in RCC. According to pathology results, 8 cases $(20.5 \%)$ were assessed as Fuhrman grade I, 24 (61.5\%) as grade II and $7(18 \%)$ as grade III. It was observed that TGF- $\beta$ expression was high in 5 cases $(12.8 \%$; Fig. $8 \mathrm{~A})$, while 21 cases $(53.8 \%$ ) demonstrated low expression (Fig. 8B). Nuclear and cytoplasmic SMAD4 expression was high in 6 cases (15.4\%; Fig. 8C) and low in 20 cases (51.3\%; Fig. 8D). TIF1- $\gamma$ expression was high in 5 cases $(12.8 \%$; Fig. $8 \mathrm{E})$, while 19 cases $(48.7 \%$ ) demonstrated low expression (Fig. 8F). Immunohistochemical staining demonstrated that SMAD4 expression was associated with higher Fuhrman grade and low expression of TIF1- $\gamma$ was associated with lower tumor 
A

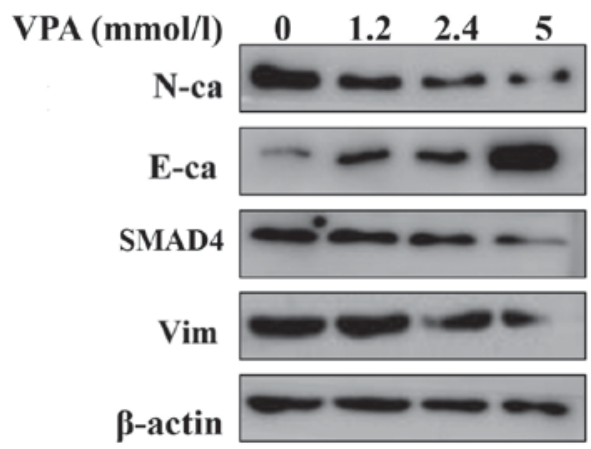

$\mathrm{C}$

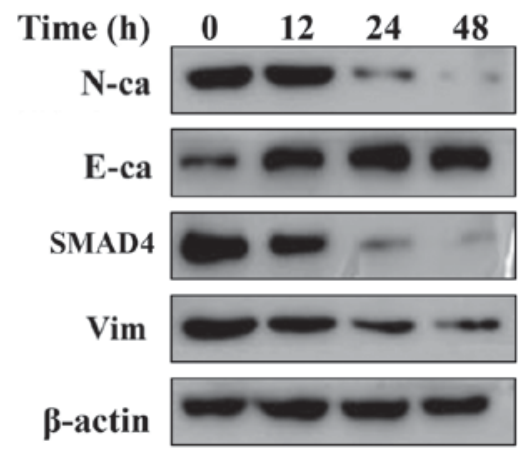

$\mathrm{B}$ 786.O dose-dependent manner

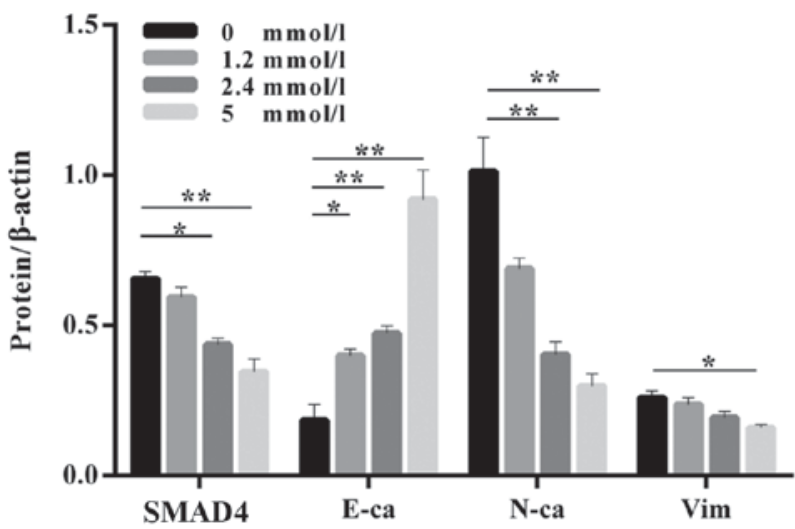

$\mathrm{D}$

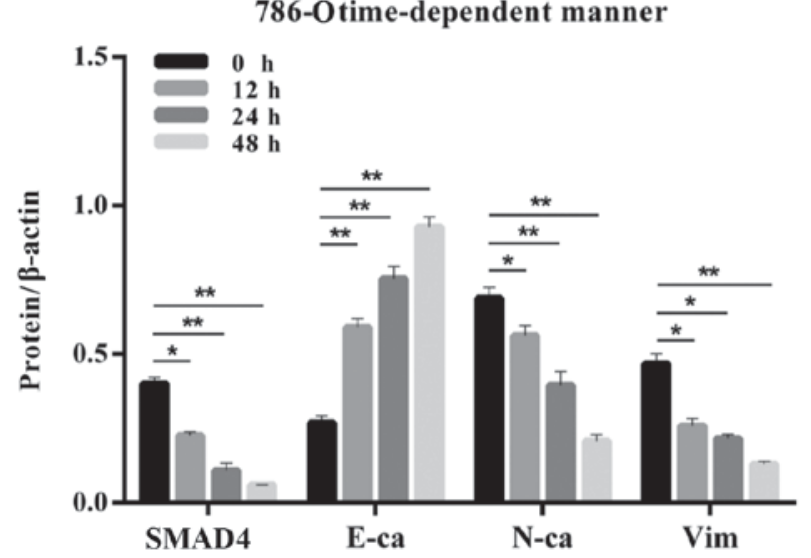

Figure 3. SMAD4 and EMT makers were inhibited by VPA in renal cell carcinoma cells. (A) Western blot and (B) quantified protein expression levels of SMAD4 and EMT makers treated with VPA in a dose-dependent manner $(0,1.2,2.4$ and $5 \mathrm{mmol} / \mathrm{l})$ in $786-\mathrm{O}$ cells. (C) Western blot and (D) quantified protein expression levels of SMAD4 and EMT makers treated with VPA in a time-dependent $(0,12,24$ and $48 \mathrm{~h})$ manner in $786-\mathrm{O}$ cells. Data is demonstrated as the mean \pm standard deviation from three independent experiments. ${ }^{*} \mathrm{P}<0.05$ vs. control group, ${ }^{* *} \mathrm{P}<0.01$ vs. control group. N-ca, neural cadherin; E-ca, epithelial cadherin; EMT, epithelial-mesenchymal transition; VPA, valproic acid; Vim, vimentin; SMAD4, Mothers against decapentaplegic homolog 4.
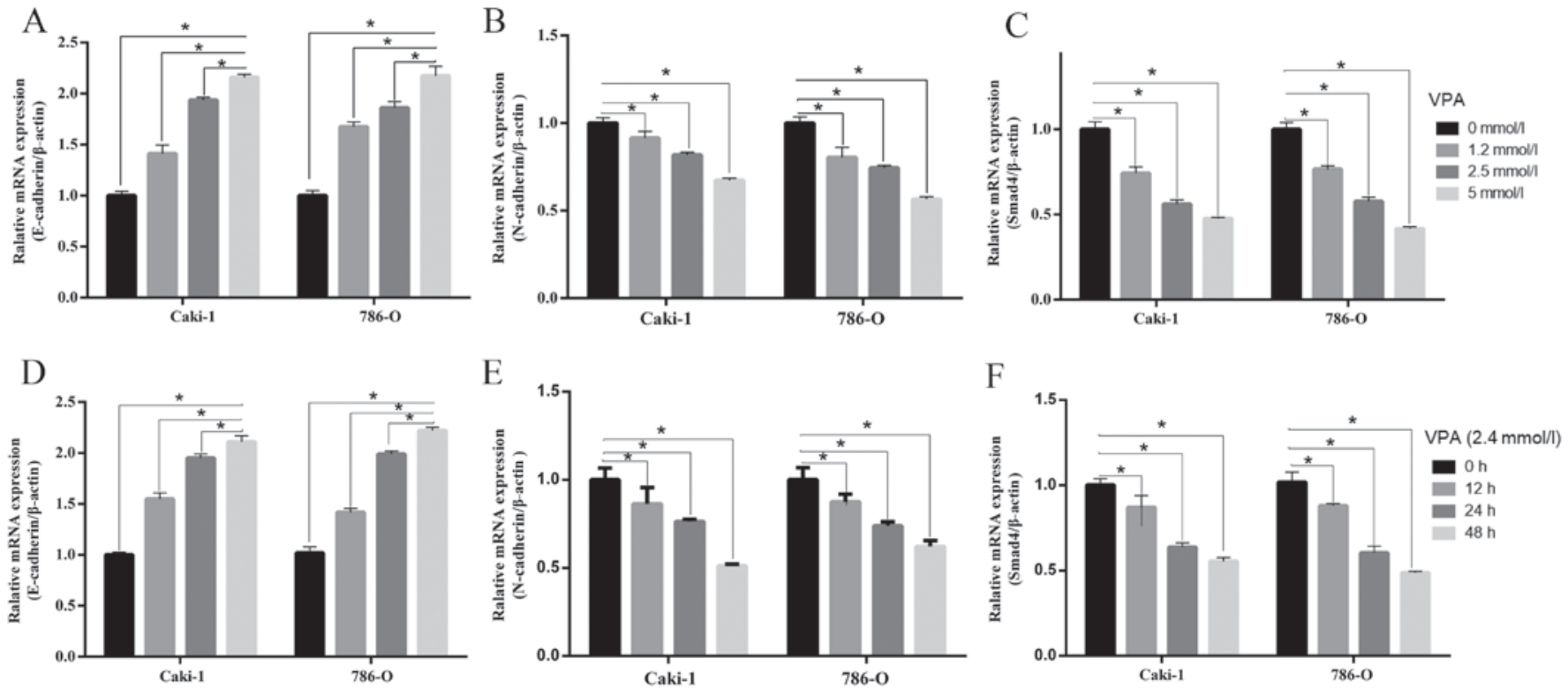

Figure 4. mRNA levels in SMAD4 and epithelial-mesenchymal transition markers. (A) E-cadherin, (B) N-cadherin and (C) SMAD4 mRNAs were measured by reverse transcription-quantitative polymerase chain reaction assay following cells treated with VPA in a dose-dependent $(0,1.2,2.4$ and $5 \mathrm{mmol} / \mathrm{l}) \mathrm{manner}$ in Caki-1 and 786-O cells. (D) E-cadherin, (E) N-cadherin and (F) SMAD4 expression following treatment with VPA in a time-dependent (0, 12, 24 and 48 h) manner in Caki-1 and 786-O cells. Data is demonstrated as the mean \pm standard deviation from three independent experiments. " $\mathrm{P}<0.05$ vs. control group, ${ }^{* *} \mathrm{P}<0.01$ vs. control group. $\mathrm{N}$-cadherin, neural cadherin; E-cadherin, epithelial cadherin; VPA, valproic acid; SMAD4, Mothers against decapentaplegic homolog 4. 
A

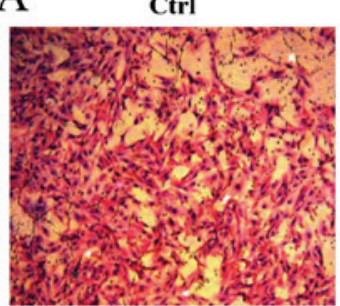

$2.4 \mathrm{mmol} / \mathrm{l}$

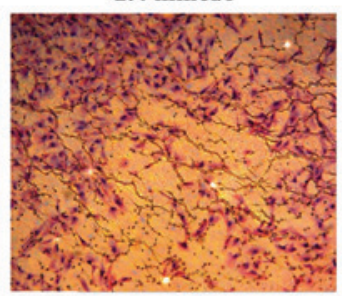

$\mathrm{C}$

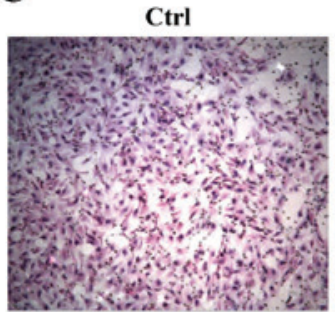

$2.4 \mathrm{mmol} / \mathrm{l}$

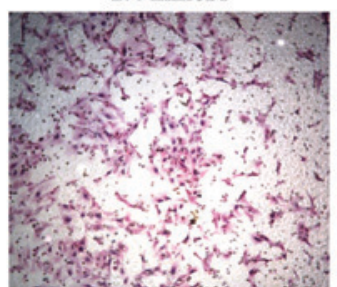

$1.2 \mathrm{mmol} /$

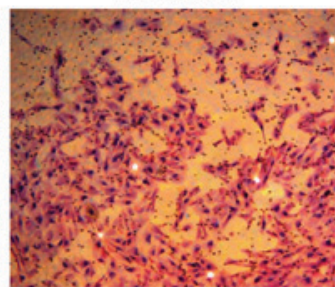

$5 \mathrm{mmol} / \mathrm{l}$

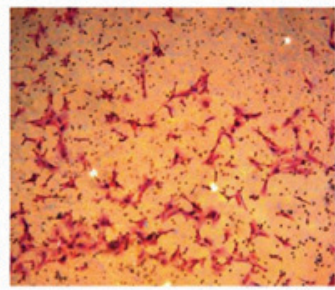

$1.2 \mathrm{mmol} / \mathrm{l}$

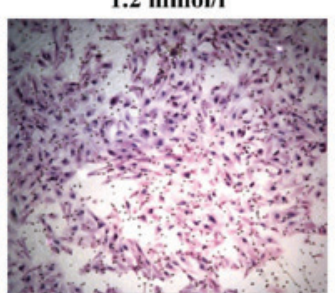

$5 \mathrm{mmol} / \mathrm{l}$

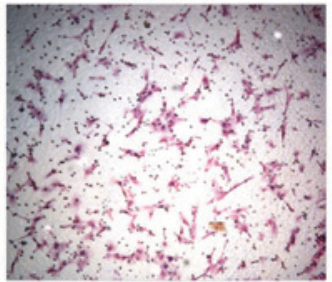

B

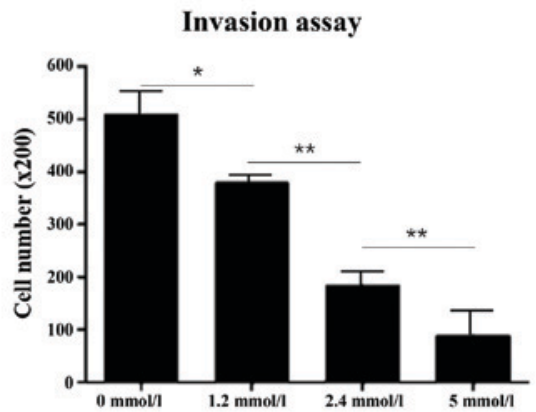

$\mathrm{D}$

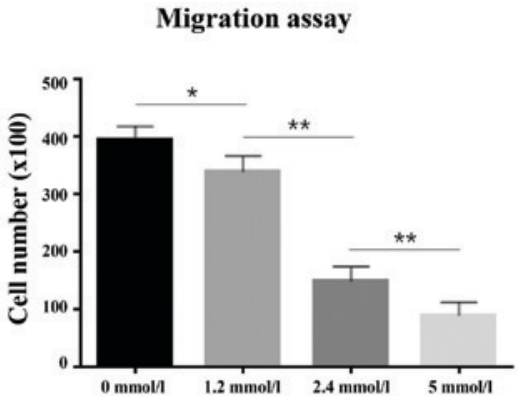

Figure 5. Analysis of the effect of VPA on the invasive and migratory ability of 786-O cells. (A) Cell invasion ability was suppressed by VPA (1.2, 2.4 and $5 \mathrm{mmol} / \mathrm{l}$ ), representative images were captured for 786-O cell line (magnification, x200) and the (B) average number of invading cells was counted. (C) Cell migration was inhibited treated by VPA $(1.2,2.4$ and $5 \mathrm{mmol} / \mathrm{l})$, representative images are demonstrated for 786-O cell line (magnification, $\mathrm{x} 100)$ and the (D) average migrating cell counts were counted. Data is demonstrated as the mean \pm standard deviation from three independent experiments. "P $<0.05$ vs. control group, ${ }^{* *} \mathrm{P}<0.01$ vs. control group. VPA, valproic acid; Ctrl, control.

A

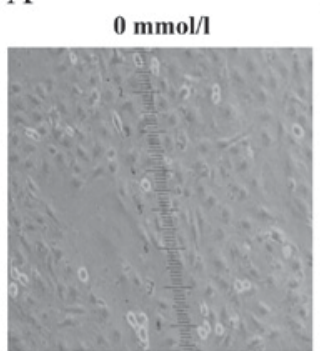

$\mathrm{E}$

$\mathbf{0 ~ h}$

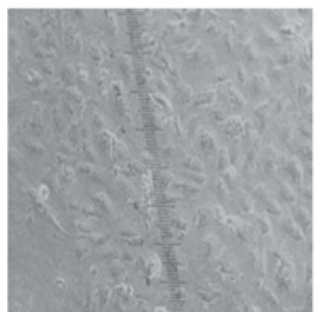

B
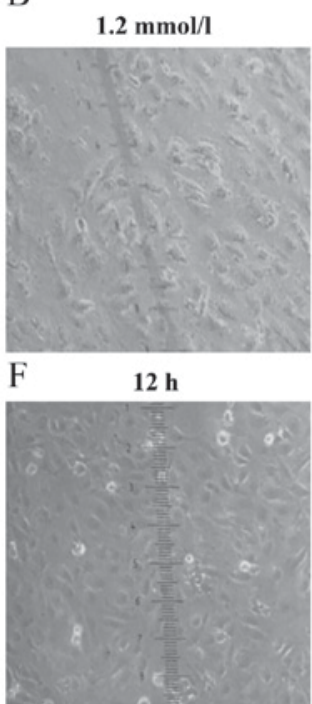

C
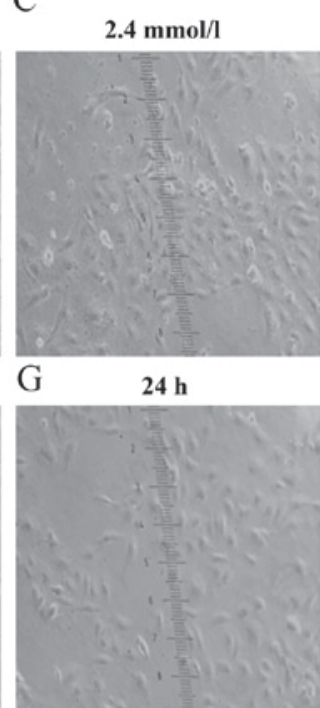

D
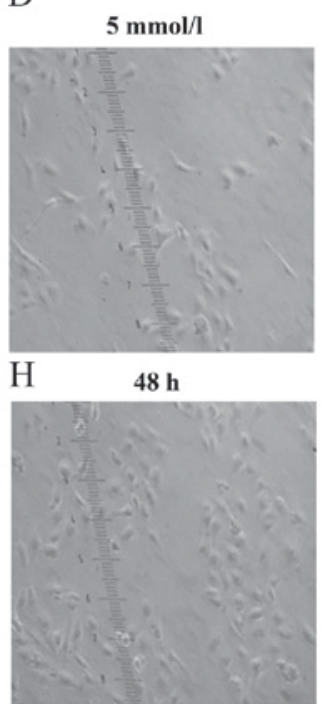

Figure 6. Images of 786-O cells treated with (A) 0, (B) 1.2, (C) 2.4 and (D) 5 mmol/1 VPA. Images of 786-O cells treated with VPA for (E) 0, (F) 12, (G) 24 and (H) 48 h. Magnification, x200. VPA, valproic acid. 
Table I. SMAD4 and TIF1- $\gamma$ expression in 39 clear renal cell carcinoma (RCC) patients with correlation with Fuhrman grade.

\begin{tabular}{|c|c|c|c|c|c|c|c|c|}
\hline \multirow[b]{2}{*}{ No. } & \multicolumn{4}{|c|}{ SMAD4 expression } & \multicolumn{4}{|c|}{ TIF1- $\gamma$ expression } \\
\hline & Low & Moderate & High & P-value & Low & Moderate & High & P-value \\
\hline Grade & & & & 0.048 & & & & 0.014 \\
\hline G I & 3 & 4 & 1 & & 5 & 3 & 0 & \\
\hline G II & 15 & 8 & 1 & & 13 & 10 & 1 & \\
\hline \multirow[t]{2}{*}{ G III } & 2 & 1 & 4 & & 1 & 2 & 4 & \\
\hline & 20 & 13 & 6 & & 19 & 15 & 5 & \\
\hline
\end{tabular}

SMAD4, Mothers against decapentaplegic homolog 4; TIF1- $\gamma$, transcription intermediary factor $1-\gamma$; TGF- $\beta$, transforming growth factor- $\beta$.


Figure 7. Western blot analysis of 786-O cells that received various treatments. (A) Western blot analysis and quantified relative protein expression levels of E-cadherin, N-cadherin, vimentin and SMAD4 expression in OE-SMAD4 786-O cells treated with $2.4 \mathrm{mmol} / \mathrm{l}$ VPA. (B) Western blot analysis and quantified relative protein expression levels of E-cadherin, N-cadherin, vimentin and SMAD4 expression in KD-SMAD4 786-O cells treated with 2.4 mmol/1 VPA. "P $<0.05$ vs. control group, ${ }^{* *} \mathrm{P}<0.01$ vs. control group. OE, over-expression; KD, knockdown; VPA, valproic acid; E-ca/E-cadherin, epithelial-cadherin; $\mathrm{N}-\mathrm{ca} / \mathrm{N}$-cadherin, neural-cadherin; Ctrl, control; SMAD4, Mothers against decapentaplegic homolog 4.

Fuhrman grade $(\mathrm{P}<0.05)$. The expression levels in the present study are listed in Table I. However, the associations between SMAD4, TIF1- $\gamma$ and TGF- $\beta$ expression and patient age, tumor size, pathological tumor, node and metastasis (pTNM) stage, cancer-specific survival or progression-free survival were not analyzed.

\section{Discussion}

Previous studies have demonstrated that EMT, a process by which epithelial cells lose their polarity and acquire a more aggressive tumor behavior, is a crucial process in the induction of tumor invasion and metastasis (19). The loss of E-cadherin expression, which is associated with the epithelial phenotype, is an event in EMT and in tumor progression, and facilitates cell invasion and metastasis, a crucial step in RCC progression (20). Early metastasis remains a challenge to the treatment of RCC. HDAC inhibitors (HDACi) have been recognized as anti-cancer agents. However, numerous other applications of HDACi have been discussed, including in infection, inflammation and innate immunity (21). HDACi have been used in renal disease, including injury by vorinostat (22) and have been used in combination with other drugs to treat RCC (23). An epidemiological phenomenon was that alterations in histone status and DNA methylation occurring in RCC induced cell proliferation and inhibited tumor growth, differentiation and apoptosis (24). Additionally, the HDACi Trichostatin A and sodium butyrate potently inhibited the development of a cancer stem cell-like phenotype in squamous cell carcinoma (25), and MS-275 and vorinostat 


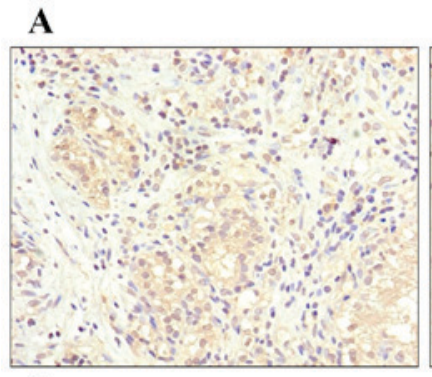

B

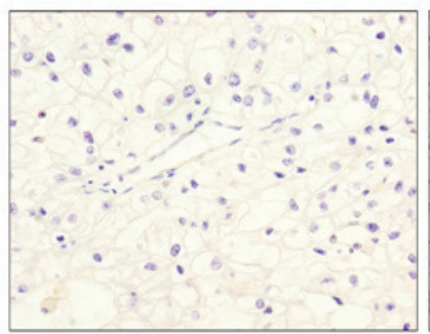

C

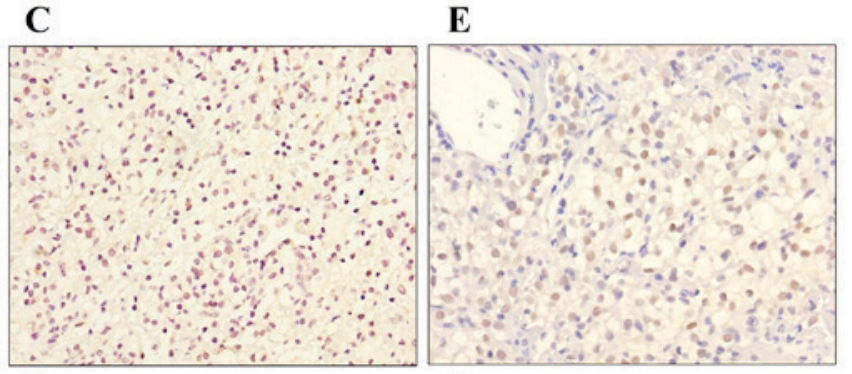

D
$\mathbf{F}$

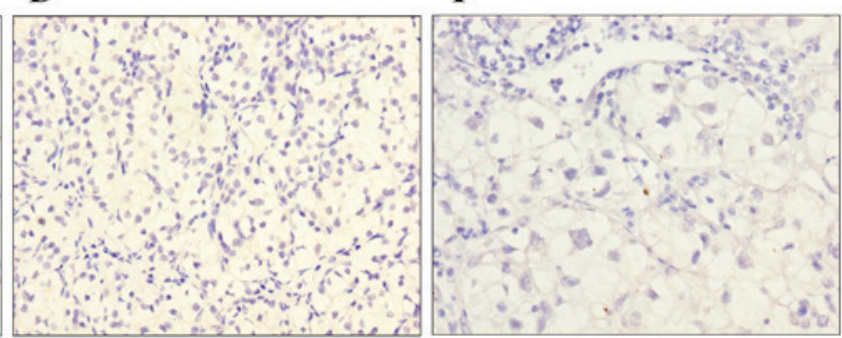

Figure 8. Representative images of immunohistochemical staining demonstrating tumor cells with (A) high and (B) low expression of TGF- $\beta$, tumor cells with (C) high and (D) low nuclear and cytoplasmic expression of SMAD4, and tumor cells with (E) high and (F) low expression of TIF1- $\gamma$. Magnification, x400 TGF- $\beta$, transforming growth factor $\beta$; TIF1- $\gamma$, transcription intermediary factor $1-\gamma ;$ SMAD4, Mothers against decapentaplegic homolog 4.

inhibited the metastatic capacity of lung and breast cancer cells (26).

The authors previously demonstrated that VPA inhibited metastasis in prostate cancer (27). The expression of E-cadherin increased following treatment with VPA in ovarian cancer (28) and this effect was further demonstrated in thyroid cancer cells (29). VPA has been used increasingly in clinical practice (30) in a novel role for the well-known drug. VPA suppresses tumor growth and metastasis (31), and also induces tumor differentiation and apoptosis in vitro and in vivo, in hematopoietic and solid malignant diseases. However, little is known about how VPA influences the invasion and metastasis of tumors. An additional study reported that HDACi could inhibit EMT (32) Based on the present study, VPA has a role in reversing EMT in RCC; VPA significantly changed EMT markers in both 786-O and Caki-1 cells. These results could provide targeted treatment in RCC.

The major control point of EMT is the TGF- $\beta$ signaling pathway and SMAD4 serves a central role in this pathway. SMAD proteins are divided into the following three subclasses: The receptor-regulated SMADs (R-SMADs), the common-partner SMADs (Co-SMAD) and the inhibitory SMADs. R-SMADs (SMAD2 and 3) bind to the Co-SMAD, SMAD4, to form complexes that modulate downstream gene expression in the nucleus (33). Previous studies have uncovered a connection between canonical SMAD4 signaling and EMT $(34,35)$. Therefore, it is necessary to test the role of SMAD4 in EMT in renal carcinoma. The present study identifies novel molecular mechanisms that may contribute to the invasion and/or metastasis of cancer cells. In the present study, 786-O cells were transfected with a plasmid knocking down SMAD4 expression. In vitro knock-down of SMAD4 resulted in increased E-cadherin levels, decreased N-cadherin expression, whereas overexpression of SMAD4 lowered E-cadherin and increased $\mathrm{N}$-cadherin expression. Invasion and metastasis are largely mediated by the loss of E-cadherin protein or functionality, as it is vital for maintaining signal transduction and preserves physical junctions in epithelial cells. E-cadherin is associated with ccRCC staging and grading as well as with lymph node involvement and the presence of distant metastasis. The epithelial or mesenchymal phenotype of cells is characterized by $\mathrm{N}$-cadherin, E-cadherin and vimentin expression. These could be targets for EMT, but to the best of the authors' knowledge no studies have been conducted into the underlying mechanism of VPA-regulation of the EMT process. In the present study, it was demonstrated that in RCC cells knockdown of SMAD4 produced the same effect as treatment with VPA: Inhibiting tumor cell invasion and migration in RCC cell lines. It was demonstrated that SMAD4 could alter $\mathrm{N}$-cadherin and E-cadherin expression, indicating a TGF- $\beta$-SMAD4-N-cadherin/E-cadherin transcription pathway. These results highlight a broad role for SMAD4 in TGF- $\beta$ induced EMT.

Despite greater understanding and identification of SMAD4 protein, it remains unclear as to what controls the switch of TGF- $\beta$ from tumor suppressor to tumor promoter. The exact function of SMAD4 has not been investigated extensively. A previous study reported that SMAD4 serves as a novel prognostic marker in patients with RCC; nuclear expression of SMAD4 was correlated with smaller tumor size, lower nuclear grade, pTNM stage and reduced tumor progression (12). Paradoxically, another study presented opposite results (33). Tumor cells usually secrete abundant TGF- $\beta$, which promotes tumor progression and the nuclear SMAD3/SMAD4 complex promotes breast cancer metastasis (36). Kang et al (33) identified that SMAD4 is critical for the TGF- $\beta$-driven upregulation of $\mathrm{N}$-cadherin and increases migration and invasion of human pancreatic ductal epithelial cells. Notably, elevation of SMAD4 is associated with poor patient outcome following surgery. Knockdown of SMAD4 reduced the efficiency of colony formation and the migratory capacity of HCC cells in vivo (37). 
The inhibitory effect of VPA on the EMT process was confirmed and the role of SMAD4 in this process was tested. However, certain problems remain to be solved. Other mechanisms may also exist that mediate the VPA-regulated EMT process. Noguchi et al (38) revealed that histone modification correlated with EMT. It is reasonable to make a connection between different media proteins and EMT regulation. TIF1 $\gamma$ may serve a potential role in cancer, targeting SMAD4 expression and cellular localization. As VPA can selectively regulate TIF1-g expression, future work should focus on TIF1- $\gamma$ and ubiquitinated SMAD4 protein, or combinational therapy.

In conclusion, the results of the present study suggest that further studies are warranted to dissect the roles of SMAD4 in tumor progression. The present study establishes the diverse mechanisms underlying cancer metastasis that involve SMAD4 regulation of EMT via the TGF- $\beta$ signaling pathway and points to potential applications of SMAD4 as an indicator of innovative, clinically effective therapies for RCC.

\section{References}

1. Ni D, Ma X, Li HZ, Gao Y, Li XT, Zhang Y, Ai Q, Zhang P, Song EL, Huang QB, et al: Downregulation of FOXO3a promotes tumor metastasis and is associated with metastasis-free survival of patients with clear cell renal cell carcinoma. Clin Cancer Res 20: 1779-1790, 2014.

2. King SC, Pollack LA, Li J, King JB and Master VA: Continued increase in incidence of renal cell carcinoma, especially in young and high-grade disease: United States 2001 to 2010. J Urol 191: 1665-1670, 2014

3. Ljungberg B, Campbell SC, Choi HY, Jacqmin D, Lee JE, Weikert S and Kiemeney LA: The epidemiology of renal cell carcinoma. Eur Urol 60: 615-621, 2011.

4. Novara G,Ficarra V, Antonelli A, Artibani W, Bertini R, Carini M, Cosciani Cunico S, Imbimbo C, Longo N, Martignoni G, et al: Validation of the 2009 TNM version in a large multi-institutional cohort of patients treated for renal cell carcinoma: Are further improvements needed? Eur Urol 58: 588-595, 2010.

5. Bertran E, Crosas-Molist E, Sancho P, Caja L, Lopez-Luque J, Navarro E, Egea G, Lastra R, Serrano T, Ramos E and Fabregat I: Overactivation of the TGF- $\alpha$ pathway confers a mesenchymal-like phenotype and CXCR4-dependent migratory properties to liver tumor cells. Hepatology 58: 2032-2044, 2013.

6. Kotiyal S and Bhattacharya S: Breast cancer stem cells, EMT and therapeutic targets. Biochem Biophys Res Commun 453: 112-116, 2014.

7. Isogaya K, Koinuma D, Tsutsumi S, Saito RA, Miyazawa K, Aburatani $\mathrm{H}$ and Miyazono K: A Smad3 and TTF-1/NKX2-1 complex regulates Smad4-independent gene expression. Cell Res 24: 994-1008, 2014

8. Qiao Y, Shiue CN, Zhu J, Zhuang T, Jonsson P, Wright AP, Zhao C and Dahlman-Wright K: AP-1-mediated chromatin looping regulates ZEB2 transcription: New insights into $\mathrm{TNF} \alpha$-induced epithelial-mesenchymal transition in triple-negative breast cancer. Oncotarget 6: 7804-7814, 2015.

9. Voorneveld PW, Kodach LL, Jacobs RJ, Liv N, Zonnevylle AC, Hoogenboom JP, Biemond I, Verspaget HW, Hommes DW, de Rooij K, et al: Loss of SMAD4 alters BMP signaling to promote colorectal cancer cell metastasis via activation of Rho and ROCK. Gastroenterology 147: 196-208.e13, 2014.

10. Wang L, Li Y, Yang X, Yuan H, Li X, Qi M, Chang YW, Wang C, $\mathrm{Fu} \mathrm{W}$, Yang M, et al: ERG-SOX4 interaction promotes epithelial-mesenchymal transition in prostate cancer cells. Prostate 74 : 647-658, 2014

11. Chaudhry P, Fabi F, Singh M, Parent S, Leblanc V and Asselin E: Prostate apoptosis response- 4 mediates TGF- $\beta$-induced epithelial-to-mesenchymal transition. Cell Death Dis 5: e1044, 2014.

12. Park JH, Lee C, Suh JH, Chae JY and Moon KC: Nuclear expression of Smad proteins and its prognostic significance in clear cell renal cell carcinoma. Hum Pathol 44: 2047-2054, 2013.
13. Gao MJ, Li X, Huang J, Gropp GM, Gjetvaj B, Lindsay DL, Wei S, Coutu C, Chen Z, Wan XC, et al: SCARECROW-LIKE15 interacts with HISTONE DEACETYLASE19 and is essential for repressing the seed maturation programme. Nat Commun 6: $7243,2015$.

14. Musselman CA, Lalonde ME, Côté J and Kutateladze TG: Perceiving the epigenetic landscape through histone readers. Nat Struct Mol Biol 19: 1218-1227, 2012.

15. Tassara M, Döhner K, Brossart P, Held G, Götze K, Horst HA, Ringhoffer M, Köhne CH, Kremers S, Raghavachar A, et al: Valproic acid in combination with all-trans retinoic acid and intensive therapy for acute myeloid leukemia in older patients. Blood 123: 4027-4036, 2014

16. Ouyang DY, Xu LH, He XH, Zhang YT, Zeng LH, Cai JY and Ren S: Autophagy is differentially induced in prostate cancer LNCaP, DU145 and PC-3 cells via distinct splicing profiles of ATG5. Autophagy 9: 20-32, 2013.

17. Zhang $X$, Zhang $X$, Huang T, Geng J, Liu M and Zheng J: Combination of metformin and valproic acid synergistically induces cell cycle arrest and apoptosis in clear cell renal cell carcinoma. Int J Clin Exp Pathol 8: 2823-2828, 2015.

18. Livak KJ and Schmittgen TD: Analysis of relative gene expression data using real-time quantitative PCR and the 2(-Delta Delta C(T)) method. Methods 25: 402-408, 2001.

19. Lamouille S, Xu J and Derynck R: Molecular mechanisms of epithelial-mesenchymal transition. Nat Rev Mol Cell Biol 15: 178-196, 2014.

20. Chen HN, Yuan K, Xie N, Wang K, Huang Z, Chen Y, Dou Q, Wu M, Nice EC, Zhou ZG and Huang C: PDLIM1 stabilizes the e-cadherin/ $\beta$-catenin complex to prevent epithelial-mesenchymal transition and metastatic potential of colorectal cancer cells. Cancer Res 76: 1122-1134, 2016.

21. Beier UH, Akimova T, Liu Y, Wang L and Hancock WW: Histone/protein deacetylases control Foxp3 expression and the heat shock response of T-regulatory cells. Curr Opin Immunol 23: 670-678, 2011.

22. Advani A, Huang Q, Thai K, Advani SL, White KE, Kelly DJ, Yuen DA, Connelly KA, Marsden PA and Gilbert RE: Long-term administration of the histone deacetylase inhibitor vorinostat attenuates renal injury in experimental diabetes through an endothelial nitric oxide synthase-dependent mechanism. Am J Pathol 178: 2205-2214, 2011.

23. Zibelman M, Wong YN, Devarajan K, Malizzia L, Corrigan A, Olszanski AJ, Denlinger CS, Roethke SK, Tetzlaff CH and Plimack ER: Phase I study of the mTOR inhibitor ridaforolimus and the HDAC inhibitor vorinostat in advanced renal cell carcinoma and other solid tumors. Invest New Drugs 33: 1040-1047, 2015.

24. Ramakrishnan S and Pili R: Histone deacetylase inhibitors and epigenetic modifications as a novel strategy in renal cell carcinoma. Cancer J 19: 333-340, 2013.

25. Chikamatsu K, Ishii H, Murata T, Sakakura K, Shino M, Toyoda M, Takahashi K and Masuyama K: Alteration of cancer stem cell-like phenotype by histone deacetylase inhibitors in squamous cell carcinoma of the head and neck. Cancer Sci 104: $1468-1475,2013$

26. Bruzzese F, Leone A, Rocco M, Carbone C, Piro G, Caraglia M, Di Gennaro E and Budillon A: HDAC inhibitor vorinostat enhances the antitumor effect of gefitinib in squamous cell carcinoma of head and neck by modulating ErbB receptor expression and reverting EMT. J Cell Physiol 226: 2378-2390, 2011.

27. Lan X, Lu G, Yuan C, Mao S, Jiang W, Chen Y, Jin X and Xia Q: Valproic acid (VPA) inhibits the epithelial-mesenchymal transition in prostate carcinoma via the dual suppression of SMAD4. J Cancer Res Clin Oncol 142: 177-185, 2016.

28. Shan Z, Feng-Nian R, Jie G and Ting Z: Effects of valproic acid on proliferation, apoptosis, angiogenesis and metastasis of ovarian cancer in vitro and in vivo. Asian Pac J Cancer Prev 13: 3977-3982, 2012.

29. Xu Y, Xu D, Zhu SJ, Ye B, Dong JD, Zhang YL and Zhang Y: Induction of apoptosis and autophagy in metastatic thyroid cancer cells by valproic acid (VPA). Int J Clin Exp Pathol 8: 8291-8297, 2015

30. Bilen MA, Fu S, Falchook GS, Ng CS, Wheler JJ, Abdelrahim M, Erguvan-Dogan B, Hong DS, Tsimberidou AM, Kurzrock R and Naing A: Phase I trial of valproic acid and lenalidomide in patients with advanced cancer. Cancer Chemother Pharmacol 75: 869-874, 2015. 
31. Byun SS, Kim FJ, Khandrika L, Kumar B, Koul S, Wilson S and Koul HK: Differential effects of valproic acid on growth, proliferation and metastasis in HTB5 and HTB9 bladder cancer cell lines. Cancer Lett 281: 196-202, 2009.

32. Mishra VK, Wegwitz F, Kosinsky RL, Sen M, Baumgartner R, Wulff T, Siveke JT, Schildhaus HU, Najafova Z, Kari V, et al: Histone deacetylase class-I inhibition promotes epithelial gene expression in pancreatic cancer cells in a BRD4- and MYC-dependent manner. Nucleic Acids Res: Mar 27, 2017.

33. Kang Y, Ling J, Suzuki R, Roife D, Chopin-Laly X, Truty MJ, Chatterjee D, Wang H, Thomas RM, Katz MH, et al: SMAD4 regulates cell motility through transcription of $\mathrm{N}$-cadherin in human pancreatic ductal epithelium. PLoS One 9: e107948, 2014.

34. Pan Y, Shu X, Sun L, Yu L, Sun L, Yang Z and Ran Y: miR196a5p modulates gastric cancer stem cell characteristics by targeting Smad4. Int J Oncol 50: 1965-1976, 2017.
35. Wu RS, Hong JJ, Wu JF, Yan S, Wu D, Liu N, Liu QF, Wu QW, Xie YY, Liu YJ, et al: OVOL2 antagonizes TGF- $\beta$ signaling to regulate epithelial to mesenchymal transition during mammary tumor metastasis. Oncotarget 8: 39401-39416, 2017.

36. Xue J, Lin X, Chiu WT, Chen YH, Yu G, Liu M, Feng XH, Sawaya R, Medema RH, Hung MC and Huang S: Sustained activation of SMAD3/SMAD4 by FOXM1 promotes TGF- $\beta$-dependent cancer metastasis. J Clin Invest 124: 564-579, 2014.

37. Hernanda PY, Chen K, Das AM, Sideras K, Wang W, Li J, Cao W, Bots SJ, Kodach LL, de Man RA, et al: SMAD4 exerts a tumor-promoting role in hepatocellular carcinoma. Oncogene 34: 5055-5068, 2015.

38. Noguchi S, Eitoku M, Moriya S, Kondo S, Kiyosawa H, Watanabe T and Suganuma N: Regulation of gene expression by sodium valproate in epithelial-to-mesenchymal transition. Lung 193: 691-700, 2015. 